\title{
Supernova Limits on the Cosmic Equation of State
}

\section{Citation}

Garnavich, Peter M., Saurabh Jha, Peter Challis, Alejandro Clocchiatti, Alan Diercks, Alexei V. Filippenko, Ron L. Gilliland, et al. 1998. "Supernova Limits on the Cosmic Equation of State." The Astrophysical Journal 509 (1): 74-79. https://doi.org/10.1086/306495.

\section{Permanent link}

http://nrs.harvard.edu/urn-3:HUL.InstRepos:41399837

\section{Terms of Use}

This article was downloaded from Harvard University's DASH repository, and is made available under the terms and conditions applicable to Other Posted Material, as set forth at http:// nrs.harvard.edu/urn-3:HUL.InstRepos:dash.current.terms-of-use\#LAA

\section{Share Your Story}

The Harvard community has made this article openly available.

Please share how this access benefits you. Submit a story.

\section{Accessibility}


The Astrophysical Journal, 509:74-79, 1998 December 10

(C) 1998. The American Astronomical Society. All rights reserved. Printed in U.S.A.

\title{
SUPERNOVA LIMITS ON THE COSMIC EQUATION OF STATE
}

\author{
Peter M. Garnavich, ${ }^{1}$ Saurabh Jha, ${ }^{1}$ Peter Challis, ${ }^{1}$ Alejandro Clocchiatti, ${ }^{2}$ Alan Diercks, ${ }^{3}$ \\ Alexei V. Filippenko, ${ }^{4}$ Ron L. Gilliland, ${ }^{5}$ Craig J. Hogan, ${ }^{3}$ Robert P. Kirshner, ${ }^{1}$ \\ Bruno Leibundgut, ${ }^{6}$ M. M. Phillips, ${ }^{7}$ David Reiss, ${ }^{3}$ Adam G. Riess, ${ }^{4}$ \\ BRIAN P. SCHMIDT, ${ }^{8}$ ROBERT A. SCHOMMER, ${ }^{7}$ R. CHRIS SMITH, ${ }^{9}$ \\ Jason Spyromilio, ${ }^{6}$ Chris Stubbs, ${ }^{3}$ Nicholas B. SuntzefF, ${ }^{7}$ \\ JOHN TONRY, ${ }^{10}$ AND SEAN M. CARROLL ${ }^{11}$ \\ Received 1998 May 28; accepted 1998 July 10
}

\begin{abstract}
We use Type Ia supernovae studied by the High- $z$ Supernova Search Team to constrain the properties of an energy component that may have contributed to accelerating the cosmic expansion. We find that for a flat geometry the equation-of-state parameter for the unknown component, $\alpha_{x}=P_{x} / \rho_{x}$, must be less than $-0.55\left(95 \%\right.$ confidence) for any value of $\Omega_{m}$, and it is further limited to $\alpha_{x}<-0.60(95 \%$ confidence) if $\Omega_{m}$ is assumed to be greater than 0.1 . These values are inconsistent with the unknown component being topological defects such as domain walls, strings, or textures. The supernova (SN) data are consistent with a cosmological constant $\left(\alpha_{x}=-1\right)$ or a scalar field that has had, on average, an equation-of-state parameter similar to the cosmological constant value of -1 over the redshift range of $z \approx 1$ to the present. SN and cosmic microwave background observations give complementary constraints on the densities of matter and the unknown component. If only matter and vacuum energy are considered, then the current combined data sets provide direct evidence for a spatially flat universe with $\Omega_{\text {tot }}=\Omega_{m}+\Omega_{\Lambda}=0.94 \pm 0.26(1 \sigma)$.

Subject headings: cosmology: observations - cosmology: theory — supernovae: general
\end{abstract}

\section{INTRODUCTION}

Matter that clusters on the scale of galaxies or galaxy clusters is insufficient to close the universe, with conventional values near $\Omega_{m}=0.2 \pm 0.1$ (Gott et al. 1974; Carlberg et al. 1996; Lin et al. 1996; Bahcall, Fan, \& Cen 1997). Observations of distant supernovae (SNs) provide credible evidence that the deceleration rate of the universal expansion is small, which implies that the total matter density, clustered or smooth, is insufficient to create a flat geometry (Garnavich et al. 1998; Perlmutter et al. 1998). Either the universe has an open geometry or, if flat, other forms of energy are more important than matter.

Large samples of SNs analyzed by the High- $z$ Supernova Search collaboration (Riess et al. 1998a, hereafter R98a) and the Supernova Cosmology Project (Kim 1998) now suggest that the universe may well be accelerating. Matter alone

\footnotetext{
${ }^{1}$ Harvard-Smithsonian Center for Astrophysics, 60 Garden Street, Cambridge, MA 02138.

${ }^{2}$ Departmento de Astronomía y Astrophisica, Pontificia Universidad Católica, Casilla 104, Santiago 22, Chile.

${ }^{3}$ Department of Astronomy, University of Washington, Seattle, WA 98195.

${ }^{4}$ Department of Astronomy, University of California, Berkeley, CA 94720-3411.

${ }^{5}$ Space Telescope Science Institute, 3700 San Martin Drive, Baltimore, MD 21218.

${ }^{6}$ European Southern Observatory, Karl-Schwarzschild-Strasse 2, Garching, Germany.

${ }^{7}$ Cerro Tololo Inter-American Observatory, Casilla 603, La Serena, Chile.

${ }^{8}$ Mount Stromlo and Siding Spring Observatory, Private Bag, Weston Creek P.O., Australia.

${ }^{9}$ University of Michigan, Department of Astronomy, 834 Dennison, Ann Arbor, MI 48109.

${ }^{10}$ Institute for Astronomy, University of Hawaii, Manoa, HI 96822.

${ }^{11}$ Institute for Theoretical Physics, University of California, Santa Barbara, CA 93106.
}

cannot accelerate the expansion; therefore, if taken at face value the observations demand an additional energy component for the universe. While the vigorous pursuit of possible systematic effects (see, e.g., Höflich, Wheeler, \& Thielemann 1998) will be important in understanding these observations, it is instructive to see what they imply about the energy content of the universe.

The cosmological constant was revived to fill the gap between the observed mass density and the theoretical preference for a flat universe (Turner, Steigman, \& Krauss 1984; Peebles 1984), as well as to alleviate the embarrassment of a young universe with older stars (Carroll, Press, \& Turner 1992). The cosmological constant is a negative pressure component arising from nonzero vacuum energy (Weinberg 1989). It would be extraordinarily difficult to detect on a small scale, but $\Omega_{\Lambda}=1-\Omega_{m}$ could make up the difference between the matter density $\Omega_{m}$ and a flat geometry and might be detected by measurements on a cosmological scale. There are few independent observational constraints on the cosmological constant, but Falco, Kochanek, \& Muñoz (1998) estimated that $\Omega_{\Lambda}<0.7$ (95\% confidence) from the current statistics of strong gravitational lenses. If the matter density is less than $\Omega_{m} \sim 0.3$, this limit is close to preventing the cosmological constant from making a flat geometry. Further, a cosmological constant that just happens to be of the same order as the matter content at the present epoch raises the issue of "fine tuning" (Coles \& Ellis 1997). A number of exotic forms of matter that might contribute to cosmic acceleration are physically possible and viable alternatives to the cosmological constant (Frieman \& Waga 1998; Caldwell, Dave, \& Steinhardt 1998). The range of possibilities can be narrowed by using SNs because the luminosity distance not only depends on the present densities of the various energy components but also depends on their equations of state while the photons we see were in flight. Here, with some simplifying assump- 
tions, we consider the constraints that recent SN observations place on the properties of an energy component accelerating the cosmic expansion.

\section{OBSERVATIONS}

The Type Ia SNs have been analyzed by the High- $z$ Supernova Search Team and described by R98a, Garnavich et al. (1998), Schmidt et al. (1998), and Riess et al. (1998b). The full sample from R98a consists of 50 Type Ia SNs. Of these, 34 are at $z<0.2$, while the remaining 16 cover a range in redshift of $0.3<z<1.0$. Six of the high- $z$ events were analyzed using the "snapshot" method developed by Riess et al. (1998b). This innovative technique uses highquality spectra to deduce information unavailable because of a poorly sampled light curve. While the errors estimated from the snapshot method are larger than those from direct light-curve fitting, the snapshot sample provides a significant, independent set of Type Ia SNs distances.

As shown by Phillips (1993), the light-curve decline rate of Type Ia SNs is correlated with the luminosity at maximum brightness of these exploding white dwarfs. This correlation has been calibrated by Hamuy et al. (1996; the $\Delta m_{15}(B)$ method) and by Riess, Press, \& Kirshner (1995, 1996; the multicolor light curve shape, or MLCS, method that includes a correction for extinction), and both show that applying this correction to the Type Ia SNs Hubble diagram significantly reduces the scatter. Phillips et al. (1998) extended the $\Delta m_{15}(B)$ approach to include an estimate of the extinction. In R98a, an improved version of the MLCS method is presented. Here, as in R98a, we apply both MLCS and $\Delta m_{15}(B)$ (with extinction correction) techniques to the analysis to gauge the systematic errors introduced by different light-curve fitting methods.

\section{ANALYSIS}

The apparent brightness of a Type Ia SN corrected for light-curve decline rate and extinction provides an estimate of the luminosity distance, $D_{L}$, from the $K$-corrected observed magnitude, $m=M+5 \log D_{L}+25$, and the absolute magnitude, $M$, of Type Ia SNs. As described by Schmidt et al. (1998) and Carroll, Press, \& Turner (1992), the luminosity distance depends on the content and geometry of the universe in a Friedmann-RobertsonWalker cosmology:

$$
\begin{gathered}
D_{L}=\frac{c(1+z)}{H_{0} \sqrt{\left|\Omega_{k}\right|}} \sin \\
\times\left\{\sqrt{\left|\Omega_{k}\right|} \int_{0}^{z}\left[\sum_{i} \Omega_{i}\left(1+z^{\prime}\right)^{3\left(1+\alpha_{i}\right)}+\Omega_{k}\left(1+z^{\prime}\right)^{2}\right]^{-1 / 2} d z^{\prime}\right\} \\
\operatorname{sinn}(x)= \begin{cases}\sinh (x), & \text { if } \Omega_{k}>0 ; \\
x, & \text { if } \Omega_{k}=0 ; \\
\sin (x), & \text { if } \Omega_{k}<0,\end{cases}
\end{gathered}
$$

where $\Omega_{i}$ are the normalized densities of the various energy components of the universe and $\Omega_{k}=1-\sum_{i} \Omega_{i}$ describes the effects of curvature. The exponent $n=3(1+\alpha)$ defines the way each component density varies as the universe expands, $\rho \propto a^{-n}$, where $a$ is the cosmic scale factor. For example, $n$ has the value 3 for normal matter since the mass density declines proportionally to the volume. Alternative- ly, $\alpha_{i}$ is the equation-of-state parameter for component $i$ defined as the ratio of the pressure to the energy density, $\alpha_{i}=P_{i} / \rho_{i}$ (sometimes denoted in the literature as $w$ ). The relation $n=3(1+\alpha)$ is easily derived from the conservation-of-energy equation in comoving coordinates (see, e.g., eq. [15.1.21] of Weinberg 1972). In the most general case, the equation of state can vary with time in ways other than assumed here (as the sum of power laws in $1+z$ ), but we are limited by the quality and range of the $\mathrm{SN}$ observations to consider only its average effect between the present and $z<1$. The present-day value of the Hubble constant $\left(H_{0}\right)$ and the absolute magnitude of Type Ia SNs $(M)$ are primarily set by the low- $z$ sample, which allows the high- $z$ events to constrain the cosmological effects. This means that conclusions derived from Type Ia SN are independent of the absolute distance scale.

Gravitational lensing by matter distributed between the observer and SNs at high $z$ can affect the observed brightness of Type Ia SNs and induce errors in the estimate of their luminosity distances (Kantowski, Vaughan, \& Branch 1995). For realistic models of the matter distribution and $\Omega_{m}<0.5$, the most likely effect of the lensing is to make the SNs at $z=0.5$ about $2 \%$ fainter than they would appear if the matter were distributed uniformly (filled beam) as shown by Wambsganss et al. (1997). Holz \& Wald (1998) have shown that the magnitude of the effect also depends on whether the matter is distributed smoothly on galaxy scales or is clumped in MACHOS, but the error induced remains small when $\Omega_{m}<0.5$. For simplicity, our calculations consider only the filled-beam case; however, the effect of assuming the extreme case of an empty beam is shown by Holz (1998).

There are a few known, and possibly some unknown, energy components that affect $D_{L}$. Ordinary gravitating matter, $\Omega_{m}$, certainly has had some effect on the universal expansion between $z \approx 1$ and now. Since the matter density scales inversely with the volume, $\alpha_{m}=0$, and matter (baryons, neutrinos, and dark matter; formerly earth, air, and water) contributes no pressure. Radiation (fire in an earlier lexicon) $\left(\alpha_{r}=\frac{1}{3}\right)$ dominated during a period in the early universe but is negligible for $z<1$. Equation (1) shows that for nonflat models the curvature term $\Omega_{k}$ contributes to the luminosity distance like a component with $\alpha_{k}=-\frac{1}{3}$, but additional geometrical effects as prescribed by equation (2) are also important.

Other more speculative components have been proposed. A nonzero vacuum energy, $\Omega_{\Lambda}$, is a popular possibility explored by R98a for this data set. Because the vacuum energy density remains constant as the universe expands (that is, $\rho_{\Lambda} \propto a^{0}$ ), we have $\alpha_{\Lambda}=-1$. Topological defects created in the early universe could also leave remnants that might contribute to the energy now. Networks of cosmic strings may be a natural consequence of phase transitions in the young universe, and if they did not intercommute they would have an average effective $\alpha_{s}=-\frac{1}{3}$ (Vilenkin 1984; Spergel \& Pen 1997). A network of comoving domain walls would have an average equation-of-state parameter of $-\frac{2}{3}$ (Vilenkin 1985), while a globally wound texture would produce $\alpha_{t}=-\frac{1}{3}$ (Davis 1987; Kamionkowski \& Toumbas 1996).

Evolving cosmic scalar fields with suitable potentials could produce a variety of exotic equations of state with significant densities at the present epoch (Peebles \& Ratra 1988; Frieman et al. 1995; Frieman \& Waga 1998). Scalar 
fields could also produce variable mass particles (VAMPs) that would redshift more slowly than ordinary matter, which would create an effective $\alpha_{\text {VAMP }}<0$ (Anderson \& Carroll 1998). These fields may evolve over time and would produce an interesting variety of cosmic histories. Our goal is modest: we only hope to constrain the average $\alpha$ over the range where Type Ia SNs are presently observed.

To simplify the analysis, we assume that only one component in addition to gravitating matter affects the cosmic expansion. Because the origin of the acceleration is unknown, we will refer to this as the $x$-component with a density of $\Omega_{x}$ and an equation of state of $P_{x}=\alpha_{x} \rho_{x}$. Caldwell et al. (1998) have dubbed the unknown component "quintessence" since the other four essences have already been employed above. We assume that the universe on very large scales is accurately described by general relativity and that the $x$-component obeys the null energy condition (NEC). The NEC states that, for any null vector $v^{\mu}$, the energy-momentum tensor satisfies $T_{\mu v} v^{\mu} v^{v} \geq 0$ (see, e.g., Wald 1984). This is the weakest of all conventional energy conditions and should be satisfied by any classical source of energy and momentum including those discussed above. In a Robertson-Walker metric, the NEC is equivalent to requiring $\rho_{x}+P_{x} \geq 0$. The NEC therefore restricts the energy density of the unknown component to be positive for $\alpha_{x}>-1$ and negative when $\alpha_{x}<-1$, while the energy density of the cosmological constant $\left(\alpha_{x}=-1\right)$ is unconstrained.

\section{RESULTS}

First, we fix the equation of state of the unknown component and estimate the probability density function for the parameters $\Omega_{x}, \Omega_{m}$, and $H_{0}$ given the observed Type Ia SNs distance moduli. The joint likelihood distributions are then calculated in the same way as by R98a and are shown for representative values of $\alpha_{x}$ in Figure 1. Here we integrate over all possible $H_{0}$ with the prior assumption that all values are equally likely. For $\alpha_{x}<-0.7$, the derived constraints are similar to those found by R98a for a cosmological constant $\left(\alpha_{x}=-1\right)$; however, as the equation-ofstate parameter increases, the major axis of the uncertainty ellipses rotates about one point on the $\Omega_{x}=0$ line. For an accelerating universe, the pivot point is on the negative $\Omega_{m}$ side. When $\alpha_{x}>-0.4$, the $x$-component could not reproduce the observed acceleration, and all of these models give a poor fit to the observed Type Ia SN data; the best fit occurs for a completely empty universe.

Next, we allow the equation of state to vary freely, but we restrict the densities to $\Omega_{m}+\Omega_{x}<1$, or open models. We then integrate over all possible values of $\Omega_{x}$ assuming a uniform prior distribution to provide the joint probability for $\alpha_{x}$ and the matter density. From the NEC we must include regions where $\alpha_{x}<-1$ and $\Omega_{x}$ is negative, but these are unable to produce an accelerating universe and therefore they have a very low probability. For open models, highest joint probabilities are confined to a region bounded

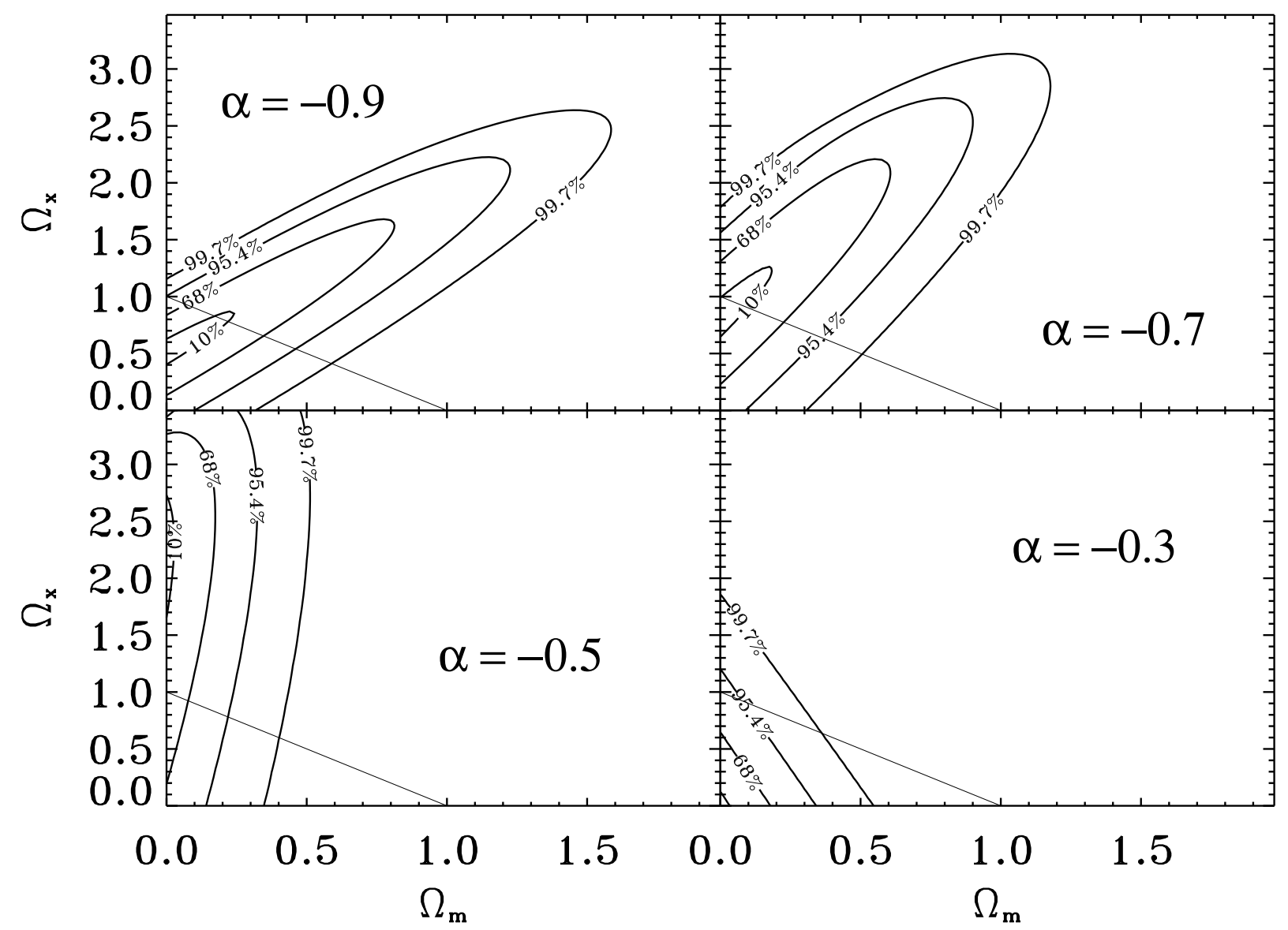

FIG. 1.- Joint probability distributions for $\Omega_{m}$ and the density of the unknown component, $\Omega_{x}$, based on the Type Ia SN magnitudes reduced with the MLCS method. Four representative values of the equation-of-state parameter, $\alpha_{x}$, are shown. See R98a for the distribution when $\alpha_{x}=-1$. 

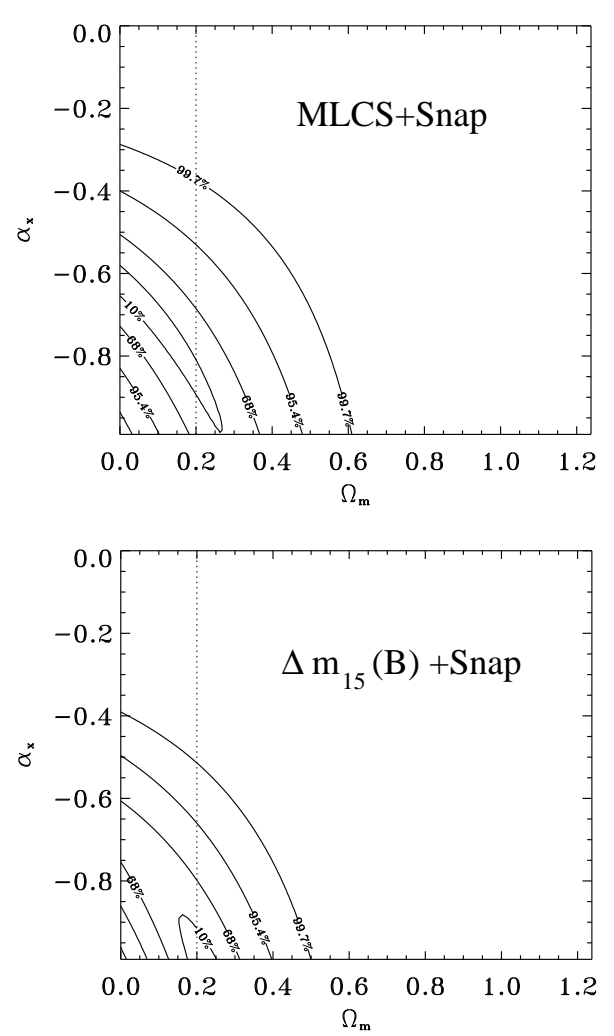

FIG. 2.- Joint probability distributions from Type Ia SNs for $\Omega_{m}$ and the equation-of-state parameter $\alpha_{x}$ assuming a flat spatial geometry $\left(\Omega_{m}+\Omega_{x}=1\right)$. The top panel uses SN distances from the MLCS method combined with SNs reduced using the snapshot method, while the bottom panel is from the $\Delta m_{15}(B)$ technique plus snapshot results. The vertical dotted line marks the matter density estimated from galaxy cluster dynamics.

by $-1.0<\alpha_{x}<-0.4$ and $\Omega_{m}<0.2$. If we consider any value of $\Omega_{m}$ equally likely, then $\alpha_{x}<-0.47$ for the MLCS method and $\alpha_{x}<-0.64$ for the $\Delta m_{15}(B)$ results with $95 \%$ confidence.

Finally, we consider flat models for the universe. The joint probability between the equation-of-state parameter and the matter density for $\Omega_{m}+\Omega_{x}=1$ is shown in Figure 2 . The two cases are for the MLCS and the $\Delta m_{15}(B)$ lightcurve fits, and they demonstrate that the two methods for deriving luminosity from light curves provide consistent constraints. Note that in the flat case, the NEC allows $\alpha_{x}<$ -1 only when $\Omega_{m}>1$ has an insignificant probability and is not plotted. These plots can be compared to pioneering calculations by Turner \& White (1997) and White (1998), which used smaller SN samples. The improved Type Ia SNs data favor acceleration and support both a low $\Omega_{m}$ and a small value of $\alpha_{x}$. Integrating the probability over all values of $\Omega_{m}$ assuming a uniform prior shows that $\alpha_{x}<-0.55$ for MLCS and $\alpha_{x}<-0.63$ for $\Delta m_{15}(B)(95 \%$ confidence). If we assume $\Omega_{m}>0.1$, then the limits tighten to $\alpha_{x}<-0.60$ (MLCS) and $\alpha_{x}<-0.69\left[\Delta m_{15}(B)\right]$. For matter densities near $\sim 0.2$ favored by galaxy cluster dispersions, the most probable equation-of-state parameters are between -0.7 and -1.0 . These results disfavor topological defect models such as domain walls ( $90 \%$ confidence) and eliminate strings and textures $(99 \%$ level) as the principal component of the unknown energy. The cosmological constant, or a form of quintessence that resembles it for $z<1$, is supported by the data. Constraints that refer to higher $z$ are needed to narrow the range of possible models.

\section{OTHER CONSTRAINTS}

High- $z$ Type Ia SNs observations combined with the cosmic microwave background (CMB) anisotropy angular power spectrum provide complementary constraints on the densities of matter and the $x$-component (White 1998; Tegmark et al. 1998). Details of the CMB power spectrum depend on a large number of variables, but the angular scale of the first acoustic peak primarily depends on the physics of recombination and the angular diameter distance to the surface of last scattering (Kamionkowski, Spergel, \& Sugiyama 1994; White \& Scott 1996; White 1998). Rather than fit the power spectrum in detail, we have restricted our attention to the location of the first acoustic peak as estimated from current CMB experiments (Hancock et al. 1998). This is a rapidly moving experimental field, and new results will surely supersede these, but they illustrate the power of combining the SN data with the CMB. We employ the analytic approximations of White (1998) to determine the wavenumber of the acoustic peak at recombination, and those of Hu \& Sugiyama (1996) to determine the recombination redshift; thus we assume adiabatic fluctuations generate the anisotropy. In addition, we have ignored reionization and fixed the number of neutrino species at three, as well as assumed only scalar modes with a spectral index of $n=1$. A thorough treatment of this problem would allow all of these parameters to vary and integrate the probability over all possible values (that is, marginalize over them); however, this would be very time consuming, even with the fast CMB code of Seljak \& Zaldarriaga (1996), and disproportionate to the precision of the current data. A large exploration of the parameter space involved (though lacking a full variation of $\Omega_{\Lambda}$ ) can be found in Bartlett et al. (1998) and Lineweaver (1998).

Our calculation determines the angular scale multipole of the first acoustic peak for a grid in a three-dimensional parameter space of $\left(\Omega_{M}, \Omega_{\Lambda}, H_{0}\right)$, where we explicitly allow for open, flat, and closed universes with and without a cosmological constant. We also employ the additional constraint on the baryon density $\Omega_{b} h^{2}=0.024\left(h=H_{0} / 100 \mathrm{~km}\right.$ $\mathrm{s}^{-1} \mathrm{Mpc}^{-1}$ ) derived from the primordial deuterium abundance and nucleosynthesis (Tytler, Fan, \& Burles 1996). Other estimates of the baryon fraction (see Fugikita, Hogan, \& Peebles 1998) could be used, but the location of the peak depends only weakly on this parameter. Where possible, we checked these calculations with numerical integrations (Seljak \& Zaldarriaga 1996) and confirmed that the peak locations agree to $\lesssim 10 \%$, which is adequate for this exploration.

Following White (1998), we combined the predicted peak location with the observations using a phenomenological model for the peak (Scott, Silk, \& White 1995). Recent CMB measurements analyzed by Hancock et al. (1998) give the conditional likelihood of the first acoustic peak position as $l_{\text {peak }}=263_{-94}^{+139}$, based on best-fit values of the peak amplitude and low-multipole normalization. Rocha et al. (1998) have provided us with a probability distribution function for the first peak position based on marginalizations over the amplitude and normalization that is a more general approach than that by Hancock et al. (1998). The Rocha et al. (1998) function gives $l_{\text {peak }}=284_{-84}^{+191}$, which is only a small shift from the value derived using the conditional likelihood method. We then marginalize the likelihood in our three-dimensional parameter space over 
$H_{0}$ with a Gaussian prior based on our own Type Ia SNs result including our estimate of the systematic error from the Cepheid distance scale, $H_{0}=65 \pm 7 \mathrm{~km} \mathrm{~s}^{-1} \mathrm{Mpc}^{-1}$ (R98a). It is important to note that the Type Ia SNs constraints on $\left(\Omega_{m}, \Omega_{\Lambda}\right)$ are independent of the distance scale but that the CMB constraints are not. We then combine marginalized likelihood functions of the CMB and Type Ia SNs data. The result is shown in Figure 3. Again, we must caution that systematic errors in either the Type Ia SNs data (R98a) or the CMB could affect this result.

Nevertheless, it is heartening to see that the combined constraint favors a location in this parameter space that has not been ruled out by other observations, though there may be mild conflict with constraints on $\Omega_{\Lambda}$ from gravitational lensing (Falco et al. 1998). In fact, the region selected by the Type Ia SN and CMB observations is in concordance with inflation, large-scale structure measurements, and the ages of stars (Ostriker \& Steinhardt 1995; Krauss \& Turner 1995). The combined constraint removes much of the high$\Omega_{m}$, high- $\Omega_{\Lambda}$ region that was not ruled out by the Type Ia SN data alone, as well as much of the high- $\Omega_{m}$, low- $\Omega_{\Lambda}$ region allowed by the CMB data alone. The combined constraint is consistent with a flat universe, as $\Omega_{\text {tot }}=\Omega_{m}$ $+\Omega_{\Lambda}=0.94 \pm 0.26$ for MLCS and $1.00 \pm 0.22$ for $\Delta m_{15}(B)$ (1 $\sigma$ errors). The enormous redshift difference between the CMB and the Type Ia SNs makes it dangerous to generalize this result beyond a cosmological-constant model because of the possible time dependence of $\alpha_{x}$. But for an equation of state fixed after recombination, the combined constraints continue to be consistent with a flat geometry as long as $\alpha_{x} \lesssim-0.6$. With better estimates of the systematic errors in the Type Ia SN data and new measurements of the CMB anisotropy, these preliminary indications should quickly turn into very strong constraints (Tegmark et al. 1998).

\section{CONCLUSIONS}

The current results from the High- $z$ Supernova Search Team suggest that there is an additional energy component sharing the universe with gravitating matter. For a flat geometry, the ratio of the pressure of the unknown energy to its density is probably more negative than -0.6 . This effectively rules out topological defects such as strings and textures as the additional component, and it disfavors domain walls as that component. Open models are less constrained but favor $\alpha_{x}<-0.5$. Although there are many intriguing candidates for the $x$-component, the current Type Ia SN observations imply that a vacuum energy or a scalar field that resembles the cosmological constant is the most likely culprit.

Combining the Type Ia SNs probability distribution with constraints of today from the position of the first acoustic peak in the CMB power spectrum provides a simultaneous

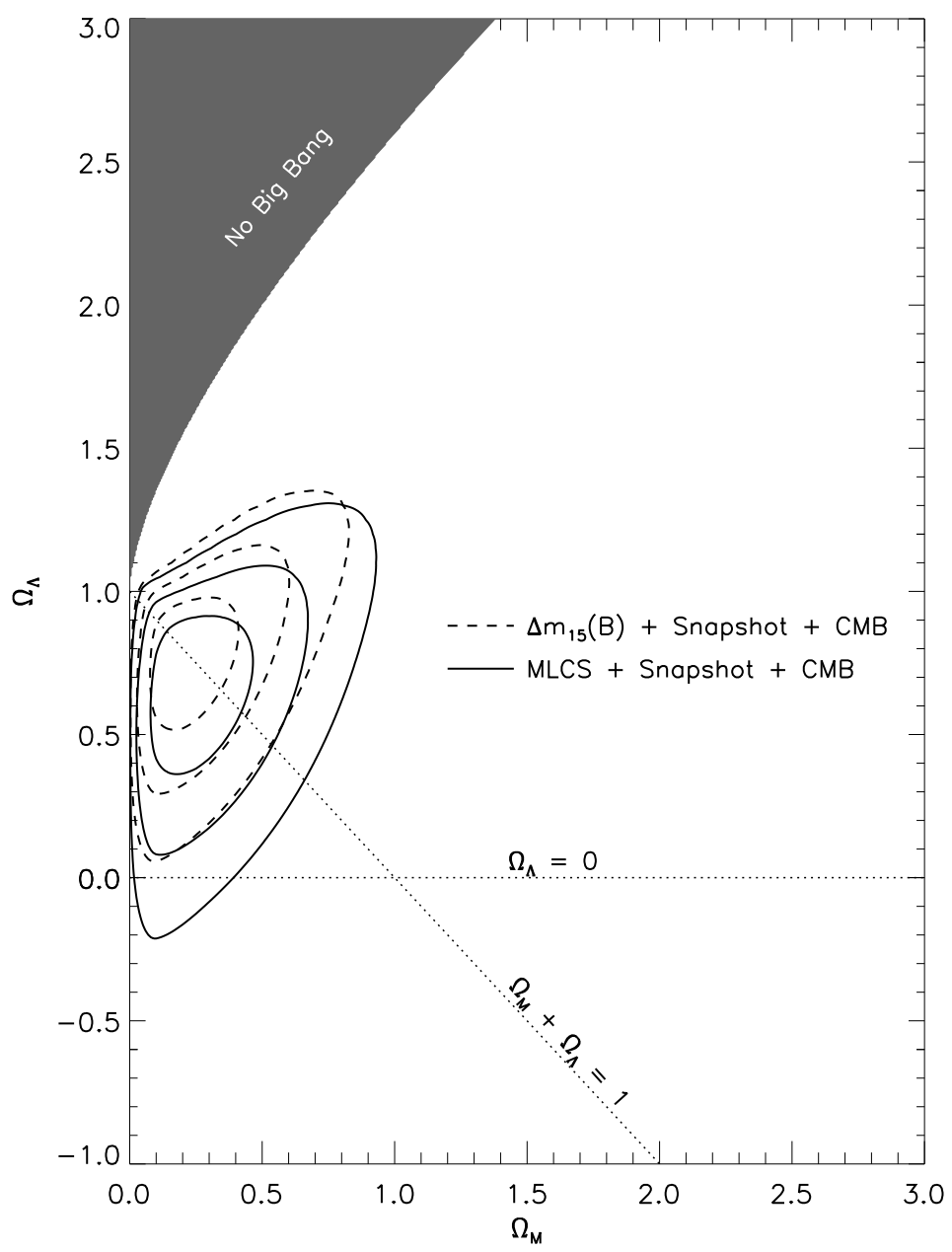

FIG. 3. - Combined constraints from Type Ia SNs and the position of the first Doppler peak of the CMB angular power spectrum. The equation-of-state parameter for the unknown component is like that for a cosmological constant, $\alpha_{x}=-1$. The contours mark the $68 \%, 95.4 \%$, and $99.7 \%$ enclosed probability regions. 
observational measurement of the densities of matter and of the unknown component. Using CMB data from Hancock et al. (1998) and following the analysis by White (1998), the result favors a flat universe with $\Omega_{\text {tot }}=0.94 \pm 0.26$, dominated by the $x$-component for $\alpha_{x} \approx-1$. Given the rapid improvement in both the study of Type Ia SNs and the $\mathrm{CMB}$, we can expect more powerful inferences about the contents of the universe to follow.

We thank U. Seljak and M. Tegmark for some informative discussions, and G. Rocha for making the CMB likeli- hood function available before publication. This work was supported by grant GO-7505 from the Space Telescope Science Institute, which is operated by the Association of Universities for Research in Astronomy, Inc., under NASA contract NAS 5-26555, and at Harvard University through NSF grants AST 92-21648 and AST 95-28899, and an NSF Graduate Research Fellowship. Work at the University of Washington was supported through NSF grant AST 9617036. A. V. F. acknowledges the support of NSF grant AST 94-17213. S. M. C. was supported by NSF grant PHY/ 94-07194.
Anderson, G. W., \& Carroll, S. M. 1998, preprint (astro-ph/9711288)

Bahcall, N. A., Fan, X., \& Cen, R. 1997, ApJ, 485, 53

Bartlett, J. G., Blanchard, A., Le Dour, M., Douspis, M., \& Barbosa, D. 1998, in Proc. of the 33d Rencontres de Moriondto, Fundamental Parameters in Cosmology, in press

Caldwell, R. R., Dave, R., \& Steinhardt, P. J. 1998, Phys. Rev. Lett., 80, 1582

Carlberg, R. G., Yee, K. C., Ellingson, E., Abraham, R., Gravel, P., Morris, S., \& Pritchet, C. J. 1996, ApJ, 462, 32

Carroll, S. M., Press, W. H., \& Turner, E. L. 1992, ARA\&A, 30, 499

Coles, P., \& Ellis, G. 1997, Is the Universe Open or Closed? (Cambridge: Cambridge Univ. Press)

Davis, R. L. 1987, Phys. Rev., 35, 3705

Falco, E. E., Kochanek, C. S., \& Muñoz, J. A. 1998, ApJ, 494, 47

Frieman, J. A., Hill, C. T., Stebbins, A., \& Waga, I. 1995, Phys. Rev. Lett., 75,2077

Frieman, J. A., \& Waga, I. 1998, Phys. Rev., 57, 4642

Fukugita, M., Hogan, C. J., \& Peebles, P. J. E. 1998, ApJ, 503, 518

Garnavich, P. M., et al. 1998, ApJ, 493, L53

Gott, J. R., Gunn, J. E., Schramm, D. N., \& Tinsley, B. M. 1974, ApJ, 194, 543

Hamuy, M., et al. 1996, AJ, 112, 2438

Hancock, S., Rocha, G., Lasenby, A. N., \& Gutiérrez, C. M. 1998, MNRAS, 294, L1

Höflich, P., Wheeler, J. C., \& Thielemann, F. K. 1998, ApJ, 495, 617

Holz, D. E. 1998, ApJ, 506, L1

Holz, D. E., \& Wald, R. 1998, Phys. Rev., D 58, 063501

Hu, W., \& Sugiyama, N. 1996, ApJ, 471, 542

Kamionkowski, M., Spergel, D. N., \& Sugiyama, N. 1994, ApJ, 426, L57

Kamionkowski, M., \& Toumbas, N. 1996, Phys. Rev. Lett., 77, 587

Kantowski, R., Vaughan, T., \& Branch, D. 1995, ApJ, 447, 35

Kim, A. 1998, in Proc. of the 33d Rencontres de Moriond, Fundamental Parameters in Cosmology, in press

Krauss, L. M., \& Turner, M. S. 1995, Gen. Relativ. Gravitation, 27, 1137

\section{REFERENCES}

Lin, H., et al. 1996, ApJ, 471, 617

Lineweaver, C. H. 1998, ApJ, submitted

Ostriker, J. P., \& Steinhardt, P. J. 1995, Nature, 377, 600

Peebles, P. J. E. 1984, ApJ, 284, 439

Peebles, P. J. E., \& Ratra, B. 1988, ApJ, 325, L17

Perlmutter, S., et al. 1998, Nature, 391, 51

Phillips, M. M. 1993, ApJ, 413, L105

Phillips, M. M., et al. 1998, in preparation

Riess, A. G., et al. 1998a, AJ, in press (R98a)

Riess, A. G., Nugent, P. E., Filippenko, A. V., Kirshner, R. P., \& Perlmutter, S. 1998b, ApJ, in press

Riess, A. G., Press, W. H., \& Kirshner, R. P. 1995, ApJ, 438, L17 1996, ApJ, 473, 88

Rocha, G., Hancock, S., Lasenby, A. N., \& Gutiérrez, C. M. 1998, in preparation

Schmidt, B. P., et al. 1998, ApJ, in press

Scott, D., Silk, J., \& White, M. 1995, Science, 268, 829

Seljak, U., \& Zaldarriaga, M. 1996, ApJ, 469, 437

Spergel, D., \& Pen, U. 1997, ApJ, 491, L67

Tegmark, M., Eisenstein, D. J., Hu, W., \& Kron, R. G. 1998, ApJ, submitted

Turner, M. S., Steigman, G., \& Krauss, L. M. 1984, Phys. Rev. Lett., 84, 2090

Turner, M. S., \& White, M. 1997, Phys. Rev., 56, 4439

Tytler, D., Fan, X.-M., \& Burles, S. 1996, Nature, 381, 207

Vilenkin, A. 1984, Phys. Rev. Lett., 53, 1016 1985, Phys. Rep., 121, 263

Wald, R. M. 1984, General Relativity (Chicago: Univ. of Chicago Press)

Wambsganss, J., Cen, R., Guohong, X., \& Ostriker, J. 1997, ApJ, 475, L81

Weinberg, S. 1972, Gravitation and Cosmology (New York: Wiley \& Sons) . 1989, Rev. Mod. Phys., 61, 1

White, M. 1998, ApJ, submitted

White, M., \& Scott, D. 1996, ApJ, 459, 415 